\title{
Exploring the Nature of Elder Abuse in Ethno-Cultural Minority Groups: A community-based participatory research study
}

\author{
Heather Haukioja* \\ The University of Victoria \\ haukioja@uvic.ca
}

\begin{abstract}
Elder abuse is a significant public health, social justice, and human rights issue in today's society. Despite the recognition that elder 1 abuse affects older adults across all racial, ethnic, and cultural groups, very little is known about the experiences of elder abuse among people from diverse ethno-cultural backgrounds in Canada. The primary objective of this study is to explore the nature of elder abuse within the two largest ethno-cultural minority groups in British Columbia (BC), the Chinese and South Asians (i.e., those who were either born in or can trace their ancestry to South Asia, which includes nations such as India, Pakistan, Sri Lanka, Bangladesh, and Nepal). Using a community-based participatory research approach, this study is a collaboration between three academics at the University of Victoria and four front-line workers from the Inter-Cultural Association of Greater Victoria (ICA), a not-for-profit, multicultural services organization for immigrants and refugees. The qualitative findings from this interview-based study reveal that cultural context, immigration status, and ethnicity are significant factors influencing experiences of elder abuse. Further, the findings provide insights into what resources - awareness and prevention - need to be developed in order to address the issue of elder abuse in these communities.
\end{abstract}

Keywords: Elder abuse; ethno-cultural minority groups; aging; culture; immigration

\section{INTRODUCTION}

$\mathrm{T}$ he demographic transition occurring in contemporary Canadian society, specifically, steady declines in fertility rates combined with rising life expectancies, has resulted in an increased aging population within Canada (McDaniel \& Tepperman, 2010). The Canadian population is projected to continue aging into the future; by 2030, close to one in four persons will be aged 65 years or older (Statistics Canada, 2014). Concurrently, Canada's population of older adults is becoming more ethno-culturally diverse due to recent immigration trends and growth among the visible minority population (Podnieks, 2008). These trends reveal the timely importance of understanding the experiences, situations, and challenges faced by older adults in various ethno-cultural communities within Canada (Podnieks, 2008). Elder abuse and neglect is one such concern and is a significant public health, social justice, and human rights issue in today's society (Dong, 2012b).

\footnotetext{
${ }^{*}$ I would like to express my sincere appreciation to Dr. Karen Kobayashi for her guidance and tremendous support throughout this project. I would also like to thank the Jamie Cassels Undergraduate Research Award for their generous support.

${ }^{1}$ The term elder is used throughout this paper to refer to older adults (i.e. those aged 65 years or older). It is used with the purpose of staying consistent with the terms used in existing academic literature and should not be conflated with the traditional Indigenous term.
} 
Generally speaking, elder abuse can be defined as a "single, or repeated act, or lack of appropriate action, occurring within any relationship where there is an expectation of trust which causes harm or distress to an older person" (WHO, 2002, p.3). The following are types of abuse that are recognized within the overall framework of elder abuse: "(1) physical abuse, which includes acts done with the intention of causing physical pain or injury; (2) psychological abuse, defined as acts done with the intention of causing emotional pain or injury; (3) sexual assault; (4) material exploitation, involving the misappropriation of the old person's money or property; and (5) neglect, or the failure of a designated carer to meet the needs of a dependent old person" (Lachs \& Pillemer, 2004, p. 1264). The effects of elder abuse on the health and quality of life of older adults are profound, including increased risks of premature morbidity and mortality (Walsh, Ploeg, Lohfeld, Horne, MacMillion, \& Lai, 2007). While it is difficult to know the exact prevalence of elder abuse within Canada due to issues of under-reporting, it is estimated that between four and ten percent of Canadian older adults experience some form of abuse or neglect from someone they trust or rely on (Public Health Agency of Canada, 2010).

Elder abuse does not discriminate; it affects older adults across all racial, ethnic, and cultural groups (Lee, Moon, \& Gomez, 2014). Despite this recognition, very little is known about the experiences of elder abuse among people from diverse ethno-cultural backgrounds in Canada (Ploeg, Lohfeld, \& Walsh, 2013). This gap in knowledge is problematic, as the limited amount of existing research indicates that ethnicity, immigration status, and cultural factors have significant influence on experiences of elder abuse, as well as on the recognition, identification, and prevention of abuse (Lai, Daoust, \& Li, 2014; Dong, 2012b). Given this knowledge, it is imperative to develop a comprehensive understanding of the nature of elder abuse within various ethno-cultural minority groups in Canada. Moreover, research is needed to identify what resources and supports must be established in order to adequately address this complex issue.

\section{Literature Review}

Elder abuse can be defined as "(a) intentional actions that cause harm or create a serious risk of harm (whether or not harm is intended), to a vulnerable elder by a caregiver or other person who stands in a trust relationship to the elder, or (b) failure by a caregiver to satisfy the elder's basic needs or to protect the elder from harm" (Bonnie \& Wallace, 2002, p.1). Although conceptualizations of what elder abuse encompasses vary, the National Center on Elder Abuse (NCEA, 2001) has identified six major forms of elder abuse, including physical abuse, sexual abuse, emotional or psychological abuse, financial abuse, neglect, and abandonment (as cited in Hafemeister, 2003). Further, there are three basic categories of elder abuse, including domestic abuse, institutional abuse, and self-neglect or self-abuse (McDonald \& Collins, 2000). The focus of this study is on domestic abuse, which is defined as any or several forms of mistreatment towards an older adult occurring in his/her own home in the community, or in a caregiver's home, by someone who has a special relationship with the older adult, such as a spouse, sibling, child, or caregiver (NCEA, 2011).

The research on elder abuse has seen significant growth since the 1980s, reaching across many different contexts (Anetzberger, 2005). Despite its increasing emergence in the literature, there remain vast gaps in our understanding of issues surrounding elder abuse. For example, conceptualizations of elder abuse within the literature often reflect a white, middle-class perspective (Moon \& Benton, 2000) while the intersections of ethnicity, cultural factors, and immigration status are largely ignored (Lai, Daoust, \& Li, 2014). In particular, scholars in the area have reviewed the literature and concluded that existing research on issues related to elder abuse in ethno-cultural minority communities is insufficient and inadequate (Lai, Daoust, \& Li, 2014; Dong, 2014). This 
is concerning as experiences such as language barriers, social isolation, financial and emotional dependency, and the characteristics of the victim and perpetrator may all be factors related to the cause and maintenance of elder abuse among ethno-cultural minority groups (Podnieks, 2008).

Another significant gap in the exploration of elder abuse is the lack of community-based participatory research (CBPR) studies in the area. It has increasingly been suggested that research partnerships between academics and community organizations be forged in order to better examine the key issues surrounding elder abuse within particular ethno-cultural minority communities (Dong, 2012a). A CBPR approach works to ensure that recognition, response, and support are offered in the most appropriate way for individuals and families with regards to abuse (Dong, 2012a). As Dong explains, using a CBPR approach allows researchers to gain a cultural awareness of the community and to develop appropriate insights surrounding prevention and intervention measures on elder abuse issues. Further, as Chang and Dong (2014) claim, by "engaging both academic and community partners in an action-driven investigation, the quality and quantity of research is enhanced without losing sight of the local community values" (p. 58). The current study is a response to these identified gaps in the existing literature as it explores the nature of elder abuse in ethno-cultural minority groups using a CBPR approach.

\section{Purpose Statement and Research Questions}

\section{Purpose Statement}

This particular study is an offshoot of a larger project currently being undertaken in British Columbia (BC) funded by the Council to Reduce Elder Abuse (CREA). The larger project represents a collaboration between academic researchers, elder law practitioners, and not-for-profit multicultural service provider administrators and staff. The CREA project examines the issue of elder abuse among ethno-cultural minority communities in the Lower Mainland, which covers the southwest corner of BC as well as in Greater Victoria.

Branching off from the larger project funded by CREA, the primary objective of this CBPR study was to explore the nature of elder abuse as it is/has been experienced by Chinese and South Asian (i.e., those who were either born in or can trace their ancestry to South Asia, which includes nations such as India, Pakistan, Sri Lanka, Bangladesh, and Nepal) older adults residing in Greater Victoria. These two particular ethno-cultural minority groups were chosen for this project as they make up the two largest ethno-cultural communities in Canada and their populations are continuing to grow rapidly within BC. As part of the larger project, this study represents a collaborative effort between three academics at the University of Victoria and four front-line workers at the Inter-Cultural Association of Greater Victoria (ICA). The not-for-profit front-line workers at ICA are responsible for providing a wide range of services and assistance to immigrants and refugees in Canada. The front-line workers at ICA were appropriate partners for this particular project as they attend to the issues of elder abuse faced by ethno-cultural minority older adults on a daily basis and are directly involved in providing support and resources to both victims and perpetrators.

\section{Research Questions}

The research questions that guided this study were negotiated and supported by the front-line workers at ICA. They are: (1) What is the nature of elder abuse (i.e., types of abuse, prevalence of abuse, factors relating to the cause and maintenance of abuse) within the two largest ethno-cultural communities in BC (Chinese and South Asians)? (2) What prevention and response resources 
currently exist and what still needs to be developed in order to appropriately address the issue of elder abuse within ethno-cultural communities in Greater Victoria?

\section{Research Design}

\section{Conceptual Framework}

In order to explore the nature of elder abuse within ethno-cultural minority communities in Greater Victoria, this study used a combination of two overlapping conceptual frameworks, the ecological and cultural sensitivity perspectives. Together, these perspectives suggest that the consideration of various contextual and socio-cultural factors is fundamental to both gaining an understanding of the nature of elder abuse and in developing culturally-responsive resources and services to address the complexity of the issue in South Asian and Chinese communities.

\section{I.1 Ecological perspective}

The ecological perspective, developed by Bronfenbrenner (1979), focuses on the various contributions that personal, interpersonal, sociocultural, political, economic, and historical exigencies play in shaping human perceptions and behaviours (as cited in Lee, Moon, \& Gomez, 2014). The ecological perspective highlights human interdependence and reciprocity with four interrelated levels of environment, including the microsystem, the mesosystem, the exosystem, and the macrosystem (Schiamberg \& Gans, 2000; Lee, Moon \& Gomez, 2014). By taking into account all of the complex, interrelated systems in which families are embedded, this perspective offers insight into how intrafamilial processes are affected by extrafamilial circumstances or conditions (Schiamberg \& Gans, 2000). Thus, rather than understanding elder abuse as a phenomenon that relates only to abusers and victims, the ecological perspective provides a comprehensive framework "for conceptualizing the relationships of the multidimensional and contextual variables associated with elder abuse" in ethno-cultural communities (Lee, Moon \& Gomez, 2014, p. 246).

\section{I.2 Cultural sensitivity perspective}

The cultural sensitivity model highlights the significance of incorporating broader socio-cultural and historical contexts, themes, and values into the development of relevant and effective interventions (Kreuter, Lukwago, Bucholtz, Clark, \& Sanders-Thompson, 2002; Resnicow, Braithwaite, Ahluwalia, \& Baranowski, 1999, as cited in Lee, Moon, \& Gomez, 2014). It seeks to explain how "members of a target population perceive and conceptualize the cause, course, and treatment of the problem within their cultural context, as well as normative practices and beliefs" (Lee, Moon, \& Gomez, 2014, p. 247). Applied to the study of elder abuse, this perspective connects the issue to broader socio-cultural and historical factors and explains perceptions of, and responses to, elder abuse within various ethno-cultural minority communities in a manner that differs from Western, more individualistic cultural explanations (Lee, Moon, \& Gomez, 2014). This approach also provides the researcher with critical insights into culturally contextualized interventions (Lee, Moon, \& Gomez, 2014). As Schiamberg and Gans (2000) maintain, the recognition and understanding of cultural diversity within a given community is critical in the design of effective prevention and intervention programs. 


\section{Methodological Approach}

Growing interest among academics in developing new ways of studying and addressing complex health and social problems has intersected in recent years with community demands for research that is community-based, rather than simply community placed (Minkler \& Wallerstein, 2008). Community-based participatory research (CBPR) can be defined as a collaborative approach to research that necessitates partnerships between academic researchers, community organizations, and key stakeholders in the examination of a particular issue (Dong, 2012b). For the purposes of this CBPR study, the core principles that were implemented included: achieving a balance between research and action; building on the strengths, skills and resources within the community; and, distributing the findings and knowledge to all partners involved.

While great diversity exists within CBPR approaches, inherent to the model are three interconnected goals: research, education, and action (Hall, 1992, as cited in Minkler \& Wallerstein, 2008). CBPR aims to combine both knowledge and action for social change to improve the community in a sustainable manner (Minkler \& Wallerstein, 2008). As Cargo and Mercer (2008) suggest, a central strength of participatory research is the "integration of researcher's" theoretical and methodological expertise with non-academic participants' real-world knowledge and experiences into a mutually reinforcing partnership" that benefits the community of interest (as cited in Minkler \& Wallerstein, 2008, p. 6).

CBPR was an appropriate approach to use in this study for several reasons. A central aspect of CBPR is ensuring that the research topic is identified by rather than for the community (Minkler \& Wallerstein, 2008). Two researchers from the larger CREA project conducted a focus group with four front-line workers at ICA on the nature of elder abuse within the Chinese and South Asian communities in October 2015. At the conclusion of this focus group, the front-line workers articulated their desire for a more in-depth exploration into the issues of elder abuse in Greater Victoria. Branching off of the larger CREA project, I collaborated with the front-line workers and decided to conduct additional research on the salient issues that arose within the first focus group. Thus, this study emerged from the front-line workers themselves, who requested the need for further research on the issues raised in the original focus group. Additionally, by engaging ICA as partners in this research project, the validity of the findings was enhanced through the recognition and integration of the local communities' values and perspectives.

\section{Methods}

Meetings with the front-line workers at ICA served a pivotal role in fostering community engagement and directed the overall exploration into the nature of elder abuse within South Asian and Chinese communities. Throughout this study, the partners at ICA were included in an ongoing, collaborative process of relationship-building, data collection, and data sharing. Implementing these collaborative measures acknowledged and supported the transfer of expertise between partners and worked to ensure cultural appropriateness of the outcomes from the study.

\section{III.1 Sample/ research setting}

This project was conducted within the Greater Victoria area. As mentioned previously, the group with whom I collaborated were four front-line service workers at the Inter-Cultural Association of Greater Victoria. This particular group of individuals were involved as partners in the study as the need to thoroughly explore the nature of elder abuse among ethno-cultural minorities in Greater Victoria was initially identified by the members of the group themselves during the original focus group in October 2015. Indeed, the staff at ICA underscored the importance and timeliness of 
this research study for their organization in the context of an increasingly diverse older adult population and, as a result, a progressively complex caseload.

\section{III.2 Data collection/ analysis}

This study involved various levels of data collection and analysis (see Figure 1). The study began with a thematic analysis of data derived from the original focus group with South Asian and Chinese front-line workers ( $\mathrm{N}=4$ participants) working at ICA that was conducted in October 2015 by two academic researchers from the larger CREA project. The results from the thematic analysis of this original focus group were used to gain initial insights into some of the key themes that may have potentially arisen within my own research project. After the data from the preliminary focus group was analyzed, I met with the four front-line workers at ICA who participated in the first focus group, and we decided upon the topics that would guide the discussion during the ensuing focus group. Once the general topic areas were negotiated, I went back to ICA and facilitated a second focus group with the same four front-line workers. The focus group methodology was chosen as the informal group discussion encourages participants to become an active part of the process and allows "research participants to explore the issues of importance to them, in their own vocabulary, generating their own questions and pursuing their own priorities" (Kitzinger, 1995, p. 299).

I let the front-line workers structure their own responses and gave them each the opportunity to discuss what they believed to be the salient issues surrounding elder abuse within the South Asian and Chinese communities, and to identify what resources they felt they needed to best support them in their work with older adults who are experiencing abuse. Prior to the focus group interview, the four participants gave written consent for their participation and for audio recording. The interview was conducted in English and was organized at the ICA welcome centre in Victoria.

All of the recorded data from the focus groups were transcribed online. I then used a multi-step process to thematically analyze the data from both focus groups, and identified the core themes that represented the nature of elder abuse and the resources needed as outlined by ICA. All of the findings were subsequently reviewed by the principal researcher and the research assistant of the larger project. After this step was completed, I sent a written transcription to the partners at ICA to review and examine the findings as part of a member check. Although not directly involved in data entry or data analysis, the ICA participants reviewed and approved all the findings and outcomes of the study to ensure cultural responsiveness and appropriateness of the recommendations for the two communities. The reason that the partners at ICA were not directly involved in every step of the data analysis and interpretation process was primarily due to the high demands for services and support that the front-line workers were experiencing during the data analysis phase.

After gaining insights from the focus groups, I constructed a visual depiction (see Figure 2 on p. 57) of the existing and needed resources that could be used to build awareness in South Asian and Chinese communities in Greater Victoria to prevent, recognize, and respond to elder abuse. What is reflected in the visual is a condensed image of the most salient issues that emerged 
throughout the focus groups. After creating the visual, I checked in with ICA and verified that what they articulated in the interviews was adequately represented in the visual depiction.
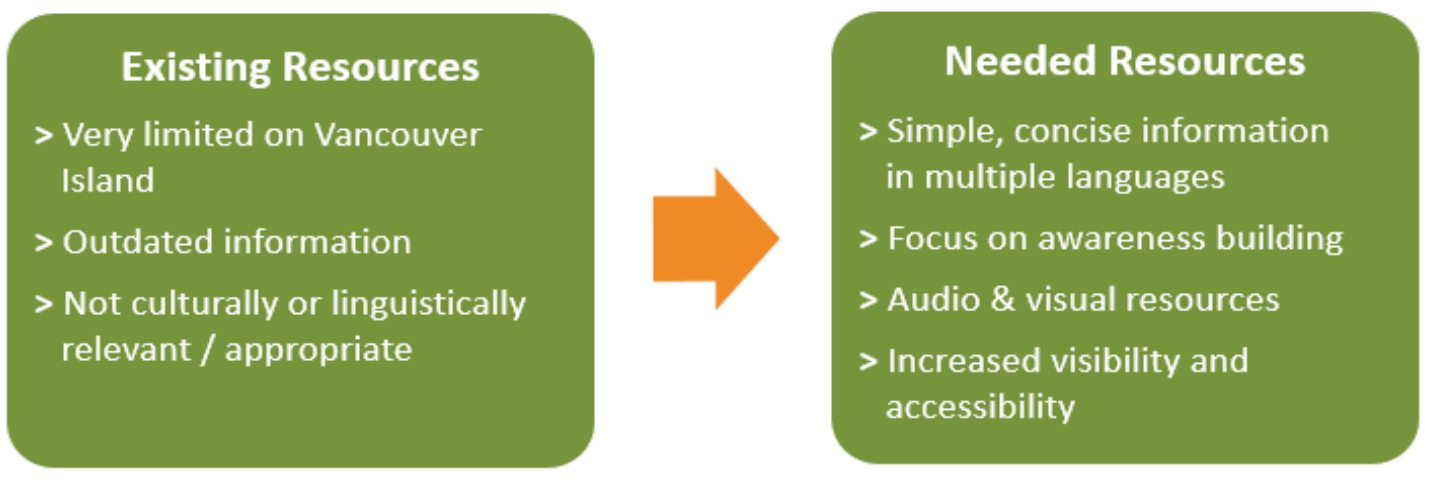

Figure 2:

Existing and Needed Resources

\section{RESEARCH FINDINGS}

\section{Participants' Characteristics}

A total of four front-line service workers from ICA participated in two sets of focus groups, which took place on October 2, 2015 and January 8, 2016. Although they held various positions at ICA, all participants were settlement workers, who were responsible for and involved in helping newcomers to Canada understand their rights and offering them assistance accessing various programs and supports. The participants were all women and all foreign-born: two were born in China and two in India. Combined, the participants spoke Cantonese, Mandarin, Vietnamese, Punjabi, Hindi, Urdu, and English. This diversity among the front-line workers was important, as many ethno-cultural minority older adults will only discuss sensitive issues with people whom they share cultural and linguistic characteristics.

\section{Definition, and Types of Elder Abuse within South Asian and Chinese Com- munities}

The front-line workers at ICA perceived elder abuse to be a salient issue in both South Asian and Chinese communities in Greater Victoria. Defined by one participant, elder abuse can be understood as "some form of harm or jeopardization of people's health and welfare" (FG1, P4). Another explained that the definition of abuse is very broad and encompasses a range of experiences (FG1, P3). When asked to describe what types of abuse the participants found to be the most prevalent in their work, all agreed that financial abuse, emotional abuse, and neglect were the most commonly experienced forms in both communities. Further, the participants were clear to mention that, in some cases, there are multiple forms of abuse occurring within a single household. When discussing how often the front-line workers encountered situations of elder abuse in their positions at ICA, one participant acknowledged that "we know that not everybody in the community is being abused... but I'll say the majority is" (FG1, P1). The participants also mentioned that there were not any significant differences between the prevalence of abuse or types of abuse experienced by South Asian as opposed to Chinese communities. 


\section{Factors Relating to the Cause and Maintenance of Elder Abuse}

\section{III.1 Social isolation}

Throughout both focus groups, the participants explained that some ethno-cultural minority older adults are socially isolated as a result of language/cultural barriers, a lack of transportation, family members' sense of control, and financial vulnerability. The participants explained that many older adults from both South Asian and Chinese communities in Greater Victoria are "very isolated at home" (FG1, P3) and have limited social networks/community connections. When asked to identify the "type" of older adult that seemed most likely to be in an abusive situation, one participant stated it was those without transportation or any means to leave the house and participate in the community (FG1, P2). These experiences of social isolation are problematic, as participants discussed how socializing outside the home and accessing various groups or programs in the community are important ways for older adults to avoid situations of abuse.

The participants also explained how social isolation, if not by choice, can be a form of abuse in and of itself. For example, family members may intentionally isolate older adults inside the home and control where they go, what they do, who they are in contact with, etc. One participant explained an incident in which the adult children "didn't give them the information like where to take English training or to socialize, to get to know new people, things like that... because 'if we let them go outside they will learn more things"' (FG1, P4). The participants interpreted this as a form of abuse, as it involved a family member hiding important information and confining the older adult to the home so that s/he would not go outside and become "aware" of their situation.

\section{III.2 Power, control, and dependency}

Power, control, and dependency were strongly emphasized by participants as underlying factors that can lead to or intensify situations of elder abuse among ethno-cultural minority older adults. The participants explained that many older adults are dependent on their family members for a number of different reasons, including language barriers, lack of available (i.e., affordable), housing in the community, lack of transportation, financial vulnerability, sponsorship policies, social isolation, health status, etc. Due to any one or a combination of these factors, many older adults are "dependent on their family" and thus "feel vulnerable to get out of the home" despite experiences of abuse (FG2, P1). In the words of one participant, these older adults "are not immersed in this culture and independently they cannot live... so that's why they sort of feel forced to live with the family" (FG2, P1).

One factor related to why relations of dependency and power imbalances between family members arises is immigration policy. The participants explained that many older adults are financially dependent as a result of Canadian sponsorship policies; that is, "because of the policy they were sponsored here, they cannot apply for subsidies for example, for rent or income assistance" (FG1, P4). Many sponsors use this dependency as a way to exert control. A number of adult children force older adults to stay home to cook, clean, and babysit, and oftentimes use threats claiming: "if you don't do what I tell you then I send you back home" (FG1, P3). The participants also explained how fears surrounding deportation or abandonment can be deterrents to sponsored older adults disclosing their experiences of abuse. For example, P3 explained a situation where a man was being physically abused by his son and when he disclosed wanting to report it to the police his wife replied, "Don't say anything... We are here because of them. Without [our] sponsor we can really not be here" (FG1). 


\section{III.3 Cultural factors}

Language Language, which is an important dimension of culture, was frequently identified as a barrier that makes discussing experiences of abuse and gaining access to services significantly more difficult for ethno-cultural minority older adults. The front-line workers explained that due to language barriers, many older adults from South Asian and Chinese communities cannot communicate with other people or understand existing information regarding how to identify and/or address experiences of elder abuse. This inability to communicate in the dominant language is identified by the participants as the most important reason why elder abuse towards ethno-cultural minority older adults is sustained. The participants all seemed to express the need for resources in the appropriate languages to help older adults identify and/or address situations of abuse. As P1 explains, although there is information about elder abuse, many of the resources are in English, which poses a significant challenge to access. Further, and perhaps of equal importance, much of this material may not be culturally relevant to ethno-cultural older adults even if it is translated into the first language of the older adult.

Cultural stigma In addition to language, the participants explained that the cultural stigma surrounding disclosure of abuse is a central reason as to why older adults from ethno-cultural minority groups do not want to discuss or report their experiences of abuse. The participants mentioned that older adults from both South Asian and Chinese communities who are experiencing various forms of abuse "don't do anything about it...it's a family reputation on the line, it's saving face" (FG1, P2). The front-line workers indicated that no matter how intense the situation of abuse is, the older adults try and cover it up due to the cultural stigma that exists and the fear that their situation will "leak out" into the community. Thus, adherence to cultural norms was an identified factor that largely influences the maintenance of abuse in South Asian and Chinese communities; it was and continues to be an issue that front-line workers face in trying to help victims of elder abuse.

Attachment to the family An additional cultural factor that influences the experience of elder abuse in ethno-cultural minority communities is the centrality of family to one's identity construction. As P3 explained, the reason why many older adults from South Asian and Chinese communities do not report situations of abuse is due to a strong attachment to the family; "they don't want to leave no matter what... they'll take [the abuse]" (FG2). One participant expanded on this point and claimed that, "even if they are dying they'll still think about the family" (FG1, P2). Moreover, the participants explained that many individuals from South Asian and Chinese communities have a fear of being separated from their family members and being placed in an institution. There is a perception that many ethno-cultural older adults would actually prefer to live with their family members despite experiences of abuse due to the strong cultural norms around the family and group harmony.

\section{III.4 Victim and perpetrator characteristics}

The participants suggested that risk factors for elder abuse also include the characteristics of both the older adult and the perpetrator. The front-line workers explained that an older adult's health status, gender, language, financial situation, amount of social support, etc. may increase their risk of being abused. For example, the participants explained how, when an older adult's mental or physical health status starts to decline, it may cause issues to arise within the family. In terms of gender, the participants indicated that being a female is a risk factor to experiences of abuse. The participants explained that while many older men are educated and engaging in the community, 
due to power relations surrounding patriarchal norms, "women are very stuck" in the home and therefore "get abused even double or more than the husband" (FG1, P1).

Regarding the characteristics of the perpetrator, the participants explained that it depends on a number of factors, such as their education, background, personality, access to resources, and caregiver burden. For example, the participants discussed caregiver burden and how some caregivers of older adults are working multiple jobs and "they are tired... they are burnt out... so they need support from other services for home support" (FG2, P2). The demands and stress placed on immigrant caregivers inside and outside the home were described by front-line workers as potentially increasing risks of elder abuse.

\section{III.5 Existing and needed resources to address the issue of elder abuse}

Existing resources When asked to describe the various resources the front-line workers at ICA currently had access to in order to help address the issue of elder abuse within the South Asian and Chinese communities, the participants responded that they had very limited resources on Vancouver Island (FG1, P3). They noted that what currently exists is outdated and not culturally or linguistically relevant to South Asian and Chinese older adults and their families. As mentioned previously, the participants explained that language barriers are the number one reason why older adults from ethno-cultural minority communities struggle to acknowledge that they are experiencing abuse - they cannot read or understand information on the issue. Thus, the participants do not end up recommending many of the existing resources to their clients at ICA as they believe that the material is not actually appropriate for them.

Needed resources In addition to existing resources, the participants were also asked to discuss what resources they believe are needed to address the issue of elder abuse among these communities in Greater Victoria. Important to note is that, although both communities are very diverse, the participants did not identify any differences between the types of resources needed for Chinese as opposed to South Asian communities. While outside the scope of this project, future in-depth research is needed to explore whether or not different resources are needed for each cultural group. The participants emphasized the need for resources that focus on increasing awareness and providing education on the issue of elder abuse. As P4 explained, they need resources that allow older adults to understand "that they have been abused, because sometimes they even don't know they are being abused" (FG2). For example, providing older adults with information regarding pension income, and explaining how family members do not have the right to take the money away would help educate older adults on their rights, and on how to recognize abuse. As one participant explained, the resources must provide the older adults with choices and information on where to go if they need help: "or saying like they have choices because they feel oh we are stuck, we don't have a choice we are helpless" (FG2, P1).

When asked what an ideal resource would look like, the participants explained that a visual resource with simple, concise information that appealed to peoples' emotions would be the most appropriate way to address issues surrounding elder abuse in ethno-cultural minority communities. The participants explained that illiteracy is a common issue for immigrant older adults and thus a visual may be easier to understand than a pamphlet or booklet because even if the older adult does not understand the words, they can see the image and relate to it.

It was underscored that resources need to be made available and accessible in multiple locations, including pharmacies, doctors' offices, the main ferry terminal, libraries, recreation centres, temples, etc. The participants further explained that awareness surrounding the issue of elder abuse should be targeted towards the whole family, not just the victim. As one participant 
explained, having a resource available to everyone is important because then the "parents can understand [what it is] and children can understand it's a crime" (FG2, P2).

\section{Discussion}

This study has provided important insights into both the nature of elder abuse among South Asian and Chinese older adults as well as guidance regarding what resources need to be developed to help address this issue in Greater Victoria. As suggested by the ecological and cultural sensitivity perspectives that guided this study, several intrafamilial and extrafamilial factors are related to the cause and maintenance of elder abuse among ethno-cultural minority groups. These include social isolation, dependency, power, and control, cultural factors, and victim and perpetrator characteristics. As articulated in the focus groups, understanding how these factors shape experiences of elder abuse among both South Asian and Chinese communities has important implications for the development of appropriate community-based prevention and intervention strategies related to the issue of elder abuse.

First, social isolation was reported by the front-line workers as a common experience among ethno-cultural minority older adults in Greater Victoria. The findings here are consistent with existing research studies, which demonstrate the impact of financial vulnerability, language, and cultural barriers, lack of transportation, power over/control from family members, etc. on the construction of situations of social isolation (Walsh et al., 2007; Tam \& Neysmith, 2006; Koehn, Spencer, \& Hwang, 2010). Social isolation is problematic, as older victims have fewer opportunities to disclose experiences of abuse and they face a number of challenges seeking out the help they need to address the issue (Alberta Elder Abuse Awareness Network, 2016). As Tam and Neysmith (2006) claim, isolation is a social condition that allows elder abuse to be effectively hidden. Further, research shows that older adults with "large social networks and connections with church, community centres, or other family members, were better able to cope in times of crisis than those with smaller social networks" (Tam \& Neysmith, 2006, p. 149).

Second, in both focus groups, participants explained how dependency and imbalances in the amount of power and control between family members are also experiences frequently faced by older ethno-cultural minority adults in Greater Victoria. The front-line workers mentioned several factors leading to South Asian and Chinese older adults' dependency on their families, including language barriers, emotional and economic vulnerability, social isolation, sponsorship arrangements, lack of understanding of their rights, etc. The findings from this study indicate that increased dependency on family members results in a heightened vulnerability to elder abuse. Further, as Chokkanathan, Natarajan, and Mohanty (2014) explain, dependency not only renders older adults susceptible to situations of abuse but may also cause older adults to feel powerless and thus more willing to endure an abusive situation and less likely to seek help.

The front-line workers highlighted the influence that Canadian sponsorship arrangements have in creating these relations of dependency and power. This is consistent with a growing body of research that recognizes how sponsorship policies may actually cause older adults to become dependent on their family members by fostering an environment of vulnerability that increases the risk of abuse in the relationship (Koehn, Spencer, \& Hwang, 2010; Ploeg, Lohfeld, \& Walsh, 2013). As mentioned by the front-line workers in this study, during the sponsorship period older adults may not be eligible for public pensions, social services, or other benefits that might foster their integration into the community and reduce their social isolation (Spencer, 2008). It is evident from the findings of this study that immigration status significantly shapes the experiences of elder abuse among South Asian and Chinese older adults in Canada.

The findings also support the need for the development and maintenance of programs and 
services that reduce the dependency of sponsored older adults on their children (Koehn, Spencer, \& Hwang, 2010). Koehn, Spencer and Hwang (2010) argue for "flexible housing arrangements (e.g. re-zoning to facilitate in-law suites), the provision of free or reduced rate bus passes for newcomers, job orientation and placement of able-bodied seniors in work that is less harmful to their health than farming, or affordable English as a Second Language classes tailored to seniors" (p. 96).

Thirdly, cultural factors play a major role in shaping Chinese and South Asian older adults' experiences of elder abuse. The participants explained that language barriers, cultural stigma, and attachment to the family are all variables that significantly impact experiences of elder abuse, particularly the help-seeking behaviours of ethno-cultural minority older adults. The participants underscored the importance of language barriers in the maintenance of abusive situations within these communities; other research has shown that language barriers can augment both dependence and isolation, making both the disclosure of abuse and the access to services significantly more challenging (Alberta Elder Abuse Awareness Network, 2016). This finding is useful in that it underscores the importance of developing resources that are linguistically and culturally relevant to people from diverse backgrounds in Canada in order to facilitate the process for older adults to report the problem and to receive the support they need.

An additional cultural factor that arose from the findings of this study is cultural stigma. The front-line workers explained that taboos against revealing private family issues may hinder disclosure of abuse; indeed many South Asian and Chinese older adults choose to remain silent about their experiences due to fear of stigma. The findings here are consistent with previous studies that indicate how silence, shame, and secrecy about elder abuse are common in many cultures (Tsukada, Saito, \& Tatara, 2001). As Chang and Dong (2014) note, the cultural stigma surrounding elder abuse can play a critical role in influencing the help-seeking behaviours of ethno-cultural minority older adults and can create significant barriers to various prevention and intervention strategies. This finding is significant as the application of a Western cultural perspective to develop an understanding of the nature of elder abuse in other cultures in Canada may not accurately account for these experiences and may lead to the development and/or implementation of inappropriate strategies to address the issue.

Fourth, the findings of this research reveal the centrality of family in the majority of South Asian and Chinese communities; many older adults will stay with their family members despite experiences of elder abuse. The participants provided insights into the fear that many ethnocultural older adults have about institutionalization and being separated from family members. Similarly, Tam and Neysmith's (2006) study on elder abuse among Chinese older adults found that "in keeping with their desire for family togetherness, Chinese seniors did not want to live in nursing homes... they would rather die than be sent to a nursing home or institution" (p. 147). This is a significant finding as it suggests that removing older adults from an abusive context and placing them in another setting may not always be the best solution to the problem due to the strong cultural preference for family togetherness. As Tam and Neysmith (2006) explain, while removing older adults from the perpetrator's home may end the abuse, it may also create a life filled with remorse and detachment for the older victim. Thus, collectivism and family harmony, as core cultural values, profoundly shape experiences of elder abuse within these two communities and directly influence help-seeking behaviour.

Finally, the characteristics of the victim and the perpetrator of elder abuse were mentioned by the front-line workers in this study as important features influencing the nature of abuse. The front-line workers expressed a wide variety of risk factors relating to the characteristics of the older adult that are consistent with other studies, including their mental / physical health status, social isolation, education, female gender, low financial resources, barriers to accessing services 
in the community, etc. (Choi \& Mayer, 2000; Lai, 2011). These risk factors associated with the characteristics of older victims provide significant insights, as specific prevention and intervention strategies should be targeted towards older adults who are at a higher risk of being neglected or abused (Lai, 2011; Dong, Simon, \& Gorbien, 2007).

Further, while the participants mentioned multiple perpetrator characteristics that influence abusive situations, caregiver burden was frequently mentioned throughout the focus groups as having an impact. This finding is consistent with other studies on the risk factors relating to elder abuse; for instance, findings from Tam and Neysmith's (2006) study reveal that many ethno-cultural minority caregivers work long hours in multiple and/or low paying jobs, and that this accumulated pressure and stress can escalate to experiences of abuse. The caregiving stress and burden felt by the caregivers can trigger situations of abuse and neglect in some cases (Choi \& Mayer, 2000; Lai, 2011; Tam \& Neysmith, 2006). This finding indicates the need for more accessible and affordable social supports for family caregivers of ethno-cultural older adults in Greater Victoria.

Overall, a central message emerging from this study is the need to understand the influence that ethnicity, cultural contexts, and immigration/sponsorship have on elder abuse and how to incorporate this knowledge into effective resources and services. Developing awareness of the issue through culturally and linguistically appropriate methods was a central step recommended by the participants to address the issue of elder abuse within these communities. Due to the academiccommunity partnership guided by CBPR principles, this study was able to gather in-depth information on a very sensitive topic and allowed for the outcomes (see Key Deliverable) to be "accessible, understandable, and locally relevant to community participants' specific interests and needs" (Leung, Yen, \& Minkler, 2004). Existing research indicates that working with communities will go a long way in ensuring that the appropriate recognition and response are developed and offered in the most suitable ways to those who experience abuse, including the victim, perpetrator, family members, and other individuals in the community (Nova Scotia Seniors' Secretariat, 2005, p. 20).

\section{Key Deliverable}

The major deliverable of this study was the visual representation of existing and needed resources identified by ICA (see Figure 2 on pg. 57). This visual was useful in condensing and mapping out the key points the participants discussed throughout both of the focus groups. Additionally, in response to the articulation of needed resources by the front-line workers at ICA, researchers on the larger study funded by CREA will develop a poster on the issue in both simplified Chinese, Punjabi, and Hindi and distribute it in places frequented by older adults and their families, including doctors' offices, pharmacies, and recreation centres. Placing the poster in a number of publicly accessible locations allows the victim, perpetrator, family members, and other individuals within the community to gain cognizance of the issue. Awareness building in the community through simple visual media was identified by the front-line workers as being an appropriate and pragmatic first response to the issue in Greater Victoria. Thus, the overall goal of the poster is to begin building awareness to prevent, recognize, and respond to elder abuse in the Chinese and South Asian communities. Further in-depth research is needed to explore what other resources and services can help address the issue of elder abuse.

The participants at ICA played a key role in identifying what should be developed based on the needs of key stakeholders in their organization and emphasized their wish that the poster be made available and accessible to all families in the identified ethno-cultural minority communities. All of the findings and outcomes of this study were verified by community partners at ICA 
as appropriately reflecting their organization's needs. Thus, the use of a community-based participatory research approach allowed for the outcomes of this study to reflect the specific interests and needs of older adults, their families, and communities.

\section{Limitations of Study}

While this study provided significant insights into the nature of elder abuse in ethno-cultural minority groups in Greater Victoria, there are limitations that are important to note. First, the sample within this study was small and was specific to one particular organization in a specific geographic location. Therefore, the extent to which the findings can be generalized to all South Asian or Chinese groups in Canada or to all front-line workers experiences is limited. Also, due to the focus group setting and the fact that all participants worked together at the same organization, the front-line workers may have been hesitant to reveal important information and may have expressed opinions that were more socially desirable answers than honest viewpoints.

Due to practical and financial reasons, data was only analyzed from two focus group interviews. Thus, further research that probes even deeper into the key issues expressed by the front-line workers would be very beneficial. Finally, although many of the guiding principles of a communitybased participatory research approach were met in this study, it was difficult to fully involve ICA in every step of the research process due to the heavy work demands the front-line workers at the organization faced during the data collection/ analysis phase of the study. Thus, in taking together all these limitations, larger CBPR studies that represent diverse ethno-cultural minority groups are certainly needed to better assess the nature of elder abuse.

\section{CONCLUSION}

Based on the findings from this study, we confirmed that elder abuse is a salient issue within the South Asian and Chinese communities in Greater Victoria. Although the issue of elder abuse has been recognized in recent decades as a serious public health and social justice concern, the intersections of ethnicity, cultural contexts, and immigration status on the nature of elder abuse have not been well researched. This gap in knowledge is extremely problematic, as this study demonstrates that these factors play a large role in shaping the experiences of elder abuse among ethno-cultural minority communities.

Further research is needed to gain in-depth understandings of the nature of elder abuse experienced by people from diverse ethno-cultural backgrounds in Canada. This research is especially important within the context of an increasingly diverse aging population in Canada. Further, it is necessary to develop culturally and linguistically appropriate resources on the recognition, prevention, and response to the issue of elder abuse. We must move away from a "one-size-fits-all" approach to dealing with the issue of elder abuse and recognize the vast diversity present within a given community.

\section{REFERENCES}

Alberta Elder Abuse Awareness Network. (2016). Ethno-Cultural Communities. from http://albertaelderabuse.ca/what-is-elder-abuse/ethno-cultural-communities.

Anetzberger, G. J. (2005). The reality of elder abuse. Clinical Gerontologist, 28(1), 1-25. http://dx.doi.org/10.1300/J018v28n01_01 
Bonnie, R. J., Wallace, R. B. (Eds.). (2003). Elder mistreatment: Abuse, neglect, and exploitation in an aging America. National Academies Press.

Bronfenbrenner, U. (1979). The Ecology of Human Development. Cambridge, MA: Harvard University Press.

Cargo, M. \& Mercer, S. L. (2008). The value and challenges of participatory research: Strengthening its practice. Annual Review of Public Health, 29, 325-350. doi:10.1146/annurev.publhealth.29.091307. 08382495-144.

Chang, E. \& Dong, X. (2014). Understanding elder abuse in the Chinese community: The role of cultural, social, and community factors. In Institute of Medicine, Forum on Global Violence Prevention, National Research Council, \& Board on Global Health, Elder Abuse and Its Prevention: Workshop Summary, (pp. 53-58). Washington (DC): National Academies Press (US). Retrieved from http:/ / www.ncbi.nlm.nih.gov/books/NBK189833/pdf/Bookshelf_NBK189833.pdf

Choi, N. G. \& Mayer, J. (2000). Elder abuse, neglect, and exploitation. Journal of Gerontological Social Work, 33(2), 5-25. doi: 10.1300/J083v33n02_02

Chokkanathan, S., Natarajan, A. \& Mohanty, J. (2014). Elder abuse and barriers to help seeking in Chennai, India: A qualitative study. Journal of Elder Abuse E Neglect, 26,(1), 60-79. doi: 10.1080/08946566.2013.782786

Dong, X., Simon, M. A. \& Gorbien, M. (2007). Elder abuse and neglect in an urban Chinese population. Journal of Elder Abuse E Neglect, 19(3-4), 79-96. doi:10.1300/J084v19n03_05

Dong, X. (2012a). Advancing the field of elder abuse: Future directions and policy implications. Journal of the American Geriatrics Society, 60(11), 2151-2516. http:/ /dx.doi.org.ezproxy.library.uvic.ca /10.1111/j.1532-5415.2012.04211.x

Dong, X. (2012b). Culture diversity and elder abuse: Implications for research, education, and policy. Generations, 36(3), 40-42. Retrieved from http://www.asaging.org/blog/culturediversity-and-elder-abuse-implications-research-education-and-policy

Dong, X. (2014). Elder abuse: Research, practice, and health policy. The 2012 GSA Maxwell Pollack Award Lecture. The Gerontologist, 54(2), 153-162. Retrieved from http://gerontologist.oxfordjournals. org $/$ citmgr?gca $=$ geront $\% 3 \mathrm{~B} 54 \% 2 \mathrm{~F} 2 \% 2 \mathrm{~F} 153$

Hall, B. L. (1992). From margins to center: The development and purpose of participatory research. American Sociologist, 23(4), 15-28.

Hafemeister, T. L. (2003). Financial abuse of the elderly in domestic setting. In R.J. Bonnie \& R.B. Wallace (Eds.), Elder Mistreatment: Abuse, Neglect, and Exploitation in an Aging America, 382-445. Washington, DC: National Academies Press. Retrieved from http://www.nap.edu. ezproxy.library.uvic.ca/read/10406/chapter/20444 
The Arbutus Review • $2016 \bullet$ Vol. 7, No. $1 \bullet$ http://dx.doi.org/10.18357/tar71201615681

Kitzinger, J. (1995). Qualitative research. Introducing focus groups. BMJ: British Medical Journal, 311(7000), 299-302. Retrieved from http://www.ncbi.nlm.nih.gov/pmc/articles/PMC2550365/

Koehn, S., Spencer, C., \& Hwang, E. (2010). Promises, promises: Cultural and legal dimensions of sponsorship for immigrant seniors. In D. Durst, M. MacLean (Eds.), Diversity and aging among immigrant seniors in Canada: Changing faces and greying temples (pp. 79-102). Calgary, AB: Detselig Enterprises Ltd.

Lachs, M. S., \& Pillemer, K. (2004). Elder abuse. The Lancet, 364(9441), 1263-1272. doi:10.1016/S0140 $-6736(04) 17144-4$

Lai, D. W. L. (2011). Abuse and neglect experienced by aging Chinese in Canada. Journal of Elder Abuse \& Neglect, 23(4), 326-327. Retrieved from http:/ / www.tandfonline.com/doi/pdf/10.1080/ 08946566.2011 .584047

Lai, D. W., Daoust, G. D. \& Li, L. (2014). Understanding elder abuse and neglect in aging Chinese immigrants in Canada. The Journal of Adult Protection, 16(5), 323-334. http:/ /dx.doi.org/10.1080/ 08946566.2011.584047

Lee, Y., Moon, A. \& Gomez, C. (2014). Elder mistreatment, culture, and help-seeking: A crosscultural comparison of older Chinese and Korean immigrants. Journal of Elder Abuse \& Neglect, 26(3), 244-269. http://dx.doi.org/10.1080/08946566.2013.820656

Leung, M. W., Yen, I. H. \& Minkler, M. (2004). Community based participatory research: A promising approach for increasing epidemiology's relevance in the 21st century. International Journal of Epidemiology, 33(3), 499-506. doi: 10.1093/ije/dyh010

McDaniel, S. A. \& Tepperman, L. (2010). Close Relations: An Introduction to the Sociology of Families (4th ed.). Toronto, ON: Pearson Canada

McDonald, L., \& Collins, A. (2000). Abuse and Neglect of Older Adults: A Discussion Paper. Ottawa, Ontario, Canada: Family Violence Prevention Unit, Health Canada.

Minkler, M. \& Wallerstein, N. (2008). Community-Based Participatory Research for Health: From Process to Outcomes. San Francisco, CA: John Wiley Sons, Publishers.

Moon, A. \& Benton, D. (2000). Tolerance of elder abuse and attitudes toward third party intervention among African American, Korean American and White elderly. Journal of Multicultural Social Work, 8(3-4), 283-303. http://dx.doi.org/10.1300/J285v08n03_05

National Centre on Elder Abuse. (2011). Major Types of Elder Abuse. Retrieved from http://www.ncea.aoa.gov/ncearoot/main_site/FAQ/Basics/Types_Of_Abuse.aspx

Nova Scotia Seniors' Secretariat. (2005). Nova Scotia Elder Abuse Strategy: Towards Awareness and Prevention. Retrieved from www.gov.ns.ca/seniorspub/2005_ElderAbuseStrategy.pdf 
The Arbutus Review • $2016 \bullet$ Vol. 7, No. $1 \bullet$ http://dx.doi.org/10.18357/tar71201615681

Ploeg, J., Lohfeld, L. \& Walsh, C. A. (2013). What is "Elder Abuse"? Voices from the margin: The views of underrepresented Canadian older adults. Journal of Elder Abuse E Neglect, 25(5), 396-424. http://dx.doi.org/10.1080/08946566.2013.780956

Podnieks, E. (2008). Elder abuse: The Canadian experience. Journal of Elder Abuse and Neglect, 20(2), 126-150. http:/ /dx.doi.org/10.1080/08946560801974612

Public Health Agency of Canada. The Chief Public Health Officer's Report on The State of Public Health in Canada 2010: The Health and Well-being of Canadian Seniors. Retrieved from http://www.phac-aspc.gc.ca/cphorsphcrespcacsp/2010/fr-rc/cphorsphcrespcacsp-06-eng. phpc36

Schiamberg, L. B. \& Gans, D. (2000). Elder abuse by adult children: An applied ecological framework for understanding contextual risk factors and the intergenerational character of quality of life. International Journal of Aging and Human Development, 50(4), 329-359.

Retrieved from http://ahd.sagepub.com.ezproxy.library.uvic.ca/content/50/4/329.full.pdf+html

Spencer, C. (2008). Immigration, abuse and capacity issues. Retrieved from http://www.bcli.org/sites/ default/files Immigration_abuse_and_capacity_issues_backgrond_paper.pdf

Statistics Canada. (2014). Population Projections: Canada, the Provinces and Territories, 2013-2063. Retrieved from http://www.statcan.gc.ca/dailyquotidien/140917/dq140917aeng.pdf

Tam, S. \& Neysmith, S. (2006). Disrespect and isolation: Elder abuse in Chinese communities. Canadian Journal on Aging, 25(2), 141-151. http:/ /dx.doi.org/10.1353/cja.2006.0043

Tsukada, N., Saito, Y. \& Tatara, T. (2001). Japanese older people's perceptions of 'elder abuse'. Journal of Elder Abuse E Neglect, 13(1), 71-89. http:/ /dx.doi.org/10.1300/J084v13n01_04

Walsh, C. A., Ploeg, J., Lohfeld, L., Horne, J., MacMillan, H. \& Lai, D. (2007). Violence across the lifespan: Interconnections among forms of abuse as described by marginalized Canadian elders and their care-givers. British Journal of Social Work, 37(3), 491-514. Retrieved from http:/ /bjsw.oxfordjournals.org/content/37/3/491.full

World Health Organization. (2002). World Report on Violence and Health (PDF). Retrieved from http://www.who.int/violence_injury_prevention/violence/world_report/en/

World Health Organization / International Network for Prevention of Elder Abuse. (2002). Missing voices: Views of older persons on elder abuse. Geneva: Authors. 